\title{
A Study of Performance Enhancement Schemes for Multicarrier Transmission
}

\author{
Chhavi Choudhary \\ Department of Wireless and Mobile Communication \\ (M.Tech.-2nd year) \\ GRD-IMT , Dehradun
}

\author{
Vishal Gupta, PhD. \\ Department of Electronics \\ GRD-IMT, Dehradun
}

\begin{abstract}
OFDM allow the transmission of high data rates over broadband channel due to the spectral bandwidth efficiency, robustness to the multipath delay etc. However OFDM signal are very sensitive to nonlinear effects due to the high peak-toaverage power radio (PAPR), which is one of the major drawback of OFDM system. The high PAPR results, high in band distortion and out-of- band radiation ,inter-carrier interference(ICI) and degradation of bit error rate (BER) performance, all these losses occur due to the non-linearity of HPA(high power amplifier). The complexity of analog-todigital converter (ADC) and digital-to- analog converter (DAC) also get increased if the PAPR of OFDM signal is high. Thus in OFDM system one of the important research areas is reduction of PAPR . This paper reviews the conventional PAPR reduction schemes for achieving the low computational complexity in wireless communication systems.
\end{abstract}

Keywords - Clipping , Companding Transform, Orthogonal frequency division multiplexing (OFDM), Partial transmit sequence(PTS), Peak-to-Average Power Ratio (PAPR), Radial basic function (RBF), Selected mapping(SLM), Tone Reservation (TR).

\section{INTRODUCTION}

OFDM has several significant advantages:

- High spectral efficiency to broadband wireless communication.

- Robustness against frequency selective fading, inter symbols interference (ISI) and narrowband interference.

- Lower implementation complexity in comparison to the single-carrier solution.

Due to these advantage OFDM (orthogonal frequency division multiplexing ) has been adopted as a standard for various wireless communication system such as digital audio broad casting(DAB),terrestrial digital video broad casting(DVB$\mathrm{T}$ ), wireless local area network(WLANs) . The main disadvantage of OFDM is its large peak-to-average power radio (PAPR) which results in significant inter-modulation and undesirable out-of band radiation. When an OFDM signal passes through high power amplifier (HPA), which is a nonlinear device [1].

In general to reduce the distortion caused by the non-linearity of HPA, it requires a large back-off from the peak power due to which the power efficiency get degrades. The complexity of the digital-to-analog converter (DAC) also get increased due to the large value of PAPR[2]. Therefore in OFDM system the PAPR reduction is one of the most important research area. There are several PAPR reduction techniques which can be classified according to some specific criteria. These PAPR techniques can be categorized as additive and multiplicative schemes with respect to the computational operation. The examples of multiplicative schemes are PTS (partial transmit sequence) and SLM (selected mapping technique) [3]. On the other hand clipping and peak canceling are deterministic schemes and tone reservation is the example of additive scheme. The low complexity PAPR reduction schemes may be applicable to mobile communication systems [4] .Comparison of PAPR reduction techniques is based on average power increase, BER degradation, computational complexity and data rate loss[5].

\section{OFDM SYSTEM MODEL}

Let $\mathrm{A}=\left[\begin{array}{lll}\mathrm{A} 0 & \mathrm{~A} 1 \mathrm{~A} 2 \ldots . . \mathrm{A} \mathrm{N}-1\end{array}\right] \mathrm{T}$ denotes an input symbol vector in the frequency domain, where $\mathrm{N}$ is the number of subcarriers and Ak represents the complex data of the kth subcarriers. The OFDM signal is generated by summing all the $\mathrm{N}$ modulated subcarriers each of which is separated by $1 / \mathrm{N}$ ts in the frequency domain, where ts represent the sampling period .Then , a continuous time OFDM signal is defined as

at $=\frac{1}{\sqrt{\mathrm{N}}} \sum_{\mathrm{k}=0}^{\mathrm{N}-1} \operatorname{Akej} 2 \pi \frac{\mathrm{k}}{\mathrm{Nts}} \mathrm{t} \quad, 0 \leq \mathrm{t}<\mathrm{Nts}$

(1)

The discrete time baseband OFDM signal an sampled at the Nyquist rate $\mathrm{t}=\mathrm{nts}$ can be given as

an $=\frac{1}{\sqrt{\mathrm{N}}} \sum_{\mathrm{k}=0}^{\mathrm{N}-1}$ Akej $2 \pi \frac{\mathrm{k}}{\mathrm{Nts}} \mathrm{n}, \quad \mathrm{n}=0,1,2 \ldots . . \mathrm{N}-1$ (2)

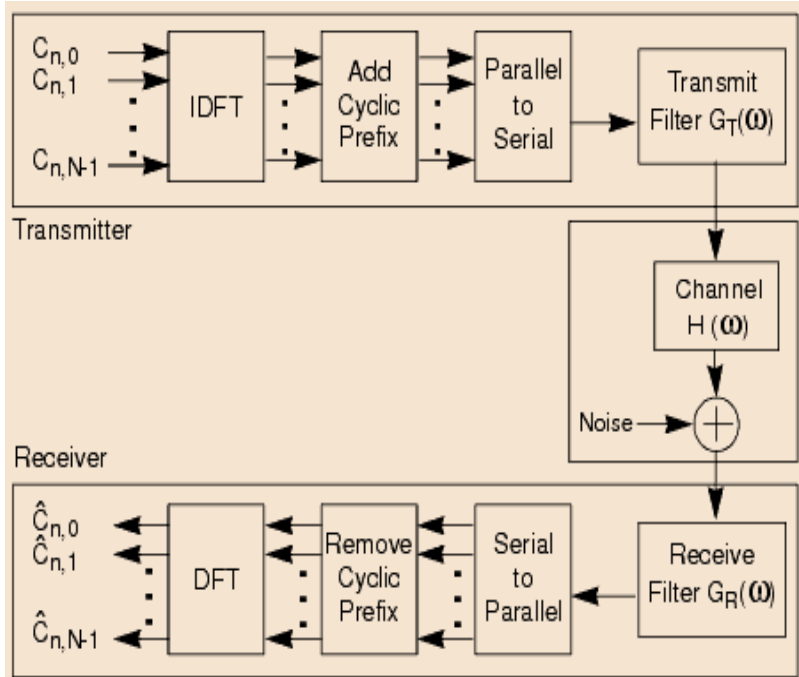

Figure-1: Block diagram of multicarrier transmission (OFDM) system 
Continuous time baseband OFDM signal can be approximately represented by $\mathrm{L}$ times oversampled discrete time baseband OFDM signals .Fig. 1 depicts the block diagram of OFDM system utilizing IDFT and filters. In this OFDM system ideal filter is used to remove a portion of out-of- band components therefore the interference problem can be reduced.

\section{PEAK-TO-AVERAGE POWER RATIO}

The PAPR of the discrete time baseband OFDM signal is defined as the ratio of the maximum peak power divided by the average power of the OFDM signal [6], that is

$\operatorname{PAPR}\left(\mathrm{a}_{\mathrm{n}}\right)=\frac{\max _{0 \leq n \leq N-1}\left|\mathrm{a}_{\mathrm{n}}\right|^{2}}{\operatorname{Pav}\left(\left|\mathrm{a}_{\mathrm{n}}\right|\right)}$

$\mathrm{P}_{\mathrm{av}}\left(\mathrm{a}_{\mathrm{n}}\right)=\frac{1}{\sqrt{N}} \sum_{n=0}^{N-1} E\left\{\left|a_{\mathrm{n}}\right|^{2}\right\}$

Where $\mathrm{E}\{$.$\} denotes the expected value.$

The PAPR of the continuous time baseband OFDM signal $a_{t}$ is defined as the ratio of the maximum instantaneous power divided by the average power of the OFDM signal, it can be expressed as

$\operatorname{PAPR}\left(\mathrm{a}_{\mathrm{t}}\right)=\frac{\max _{0 \leq n \leq N t_{s}}\left|\mathrm{a}_{\mathrm{t}}\right|^{2}}{\operatorname{Pav}\left(\left|\mathrm{a}_{\mathrm{t}}\right|\right)}$

Where $\mathrm{P}_{\mathrm{av}}\left(\mathrm{a}_{\mathrm{t}}\right)=\frac{1}{N t_{s}} \int_{0}^{N t_{s}} E\left\{\left|\mathrm{a}_{\mathrm{t}}\right|^{2}\right\} \mathrm{dt}$

And the PAPR of the continuous time pass band OFDM signal $\mathrm{g}_{\mathrm{t}}$ is also defined as

$\operatorname{PAPR}\left(\mathrm{g}_{\mathrm{t}}\right)=\frac{\max _{0 \leq n \leq N t_{s}}\left|\mathrm{~g}_{\mathrm{t}}\right|^{2}}{\operatorname{Pav}\left(\left|\mathrm{g}_{\mathrm{t}}\right|\right)}$

The discrete time baseband OFDM signals, are transformed in to continuous time baseband OFDM signals by a low pass filter called DAC, where the peak power can be increased while maintaining constant average power. Usually, the PAPR of continuous time baseband OFDM signals is larger than that of discrete time baseband OFDM signals by $0.5-1.0 \mathrm{~dB}$.[8]

\section{PAPR REDUCTION TECHNIQUES}

Various techniques have been developed to reduce the PAPR of multicarrier OFDM system, high PAPR is the major drawback in multicarrier system. In this section we have discussed different PAPR reduction techniques

\section{1 .Clipping}

The clipping is one of the easiest technique which is used to reduce the power by setting a maximum level for the transmitted signal [9]. Though, this technique has several disadvantages:

(i) Due to the in-band distortion caused by clipping technique the performance of the bit error rate (BER) may be affected (ii) Also out-of-band radiation, which usually appears with clipping technique and disturb the adjacent channels
However filtering operation can be used to decrease the appearance of the out-of-band radiation but the signal may exceed the maximum level of clipping operation. The outcome of filtering provide a less degraded BER performance.Fig.-2 shows the block diagram of clipping and filtering .The clipped form of pass band modulated signal is given as

$$
\begin{array}{lll}
-\mathrm{A} & x^{p}[\mathrm{~m}] \leq-\mathrm{A} \\
x_{c}^{p}[\mathrm{~m}]= & x^{p}[\mathrm{~m}] \\
\mathrm{A} & x^{p}[\mathrm{~m}] \geq \mathrm{A}
\end{array} \quad x^{p}[\mathrm{~m}] \mid<\mathrm{A}
$$

\subsection{Companding Transform}

Companding technique is one of the useful techniques which is used for the reduction of PAPR (peak-to-average power ratio) in an OFDM signal. In Figure-2: Block diagram of clipping technique has been shown

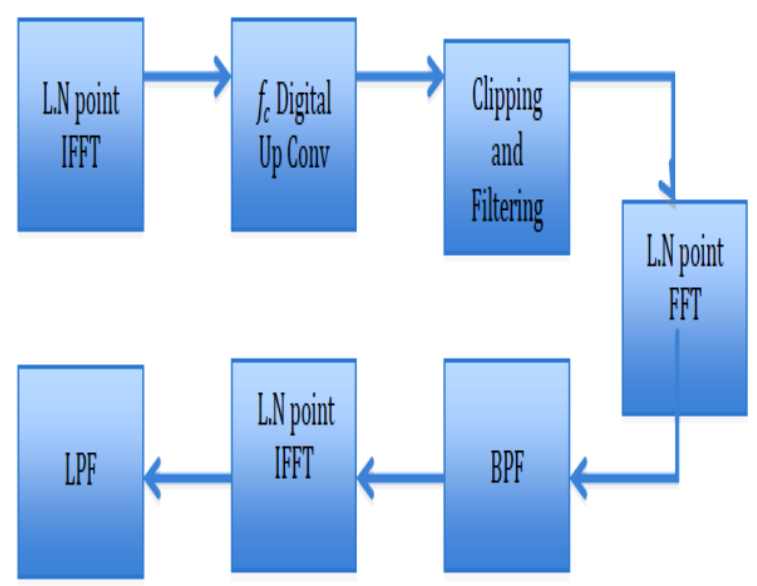

Figure-2: Block diagram of clipping.

companding transform at the transmitter end compression is used after IFFT (Inverse fast fourier transform) operation and at receiver end expansion is used before FFT (Fast fourier transform) process. For discrete OFDM signal the companded signal is given by[10]

$\mathrm{S}(\mathrm{n})=\frac{a x(n)}{\ln (1+\mu)|x(n)|} \ln \left(1+\frac{\mu}{v}|x(n)|\right.$

Where a- represent average amplitude of the signal and $\mu$ is the companding parameter.

This transform reduces the PAPR of OFDM signal by attenuating the period of high signal and by amplifying the small signals.

\subsection{Partial Transmit Sequence}

The partial transmit sequence (PTS) is a powerful PAPR reduction technique for OFDM signals .The block diagram of the PTS scheme is shown in Fig.- 3. 


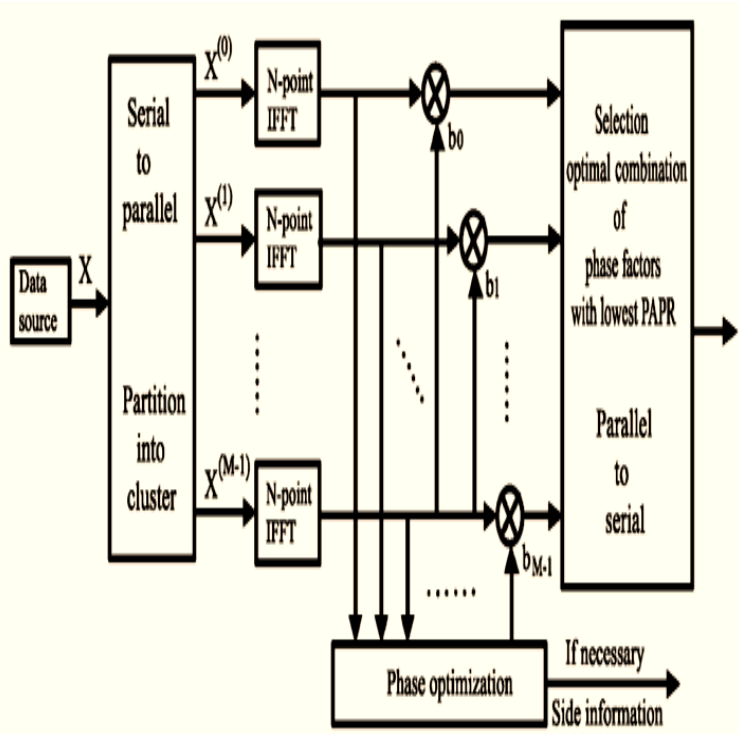

Figure-3: Block diagram of PTS technique

In the given fig.- $3, \mathrm{X}$ is the input data which is partitioned into $M$ disjoint sub-blocks. The sub-carriers in each sub-block are weighted by a phase factor for that sub-block. The phase factors are selected such that the PAPR of the combined signal is minimized[11]-[12]. The input data $\mathrm{X}$ divided into $\mathrm{M}$ disjoint sub-block is expressed as

$$
X^{m}=X_{0}^{m}, X_{1}^{m} \ldots \ldots . X_{N-1}^{m}, m=1,2 \ldots . M
$$

The original signal of sub block is given as

$\mathrm{X}=\sum_{m=1}^{M} X^{m}$

The sub-block partition techniques are of three type, named as interleaved partition, adjacent partition, and random partition. The interleaved partition has the worst PAPR reduction performance the random partition technique is the best choice for PAPR reduction of OFDM signals. To combine the PTSs the Complex phase factors are introduced. The set of phase factors is denoted as a vector

$\mathrm{b}=\left[b_{1}, b_{2 \ldots \ldots} b_{M,}\right]$

To minimize the PAPR we have to find the optimum set of phase factor as the phase factors are introduced to combine the PTSs[13]. The relationship between the phase factor and partial transmit sequence (PTS) given as

$$
\begin{gathered}
x^{\prime}=\sum_{m=1}^{M} \operatorname{IFFT}\left\{X^{m} \cdot b^{m}\right\}=\sum_{m=1}^{M} b^{m} \cdot \operatorname{IFFT}\left\{X^{m}\right\} \\
\left.x^{\prime}=\sum_{m=1}^{M} b^{m} \cdot x^{m}\right\}
\end{gathered}
$$

Where $b^{m}=\Theta, \quad \Theta=\left\{e^{j \theta_{1}}, \quad e^{j \theta_{2}} \ldots e^{j \theta_{V}}\right\}$ (14)

Here $\Theta$ represents the set which includes V phase factor and $x^{m}$ is called partial transmit sequence.

\subsection{Radial Basic Function}

As shown in Fig.-4. a radial basic function Neural Network (RBFNN) consists of three basic layers: the input layer, the hidden layer, and the output layer. In RBF from the input space to the hidden-unit space the transformation is nonlinear, whereas the mapping from the hidden-unit space to the output space is linear. The hidden layer's activation functions can be modifying according to some nonlinear optimization method and for the output layer, the weights evolve rapidly through some linear optimization strategy. Thus, different techniques can be utilized to train the different layers of the RBF neural network. The RBFN technique is an adaptive nonlinear optimum OFDM signal mapping technique that reduces the PAPR substantially. Radial basis function network (RBFN) [14] is one of the most used method which has been applied successfully in areas such as channel equalization and [15] and multiuser detection [16].

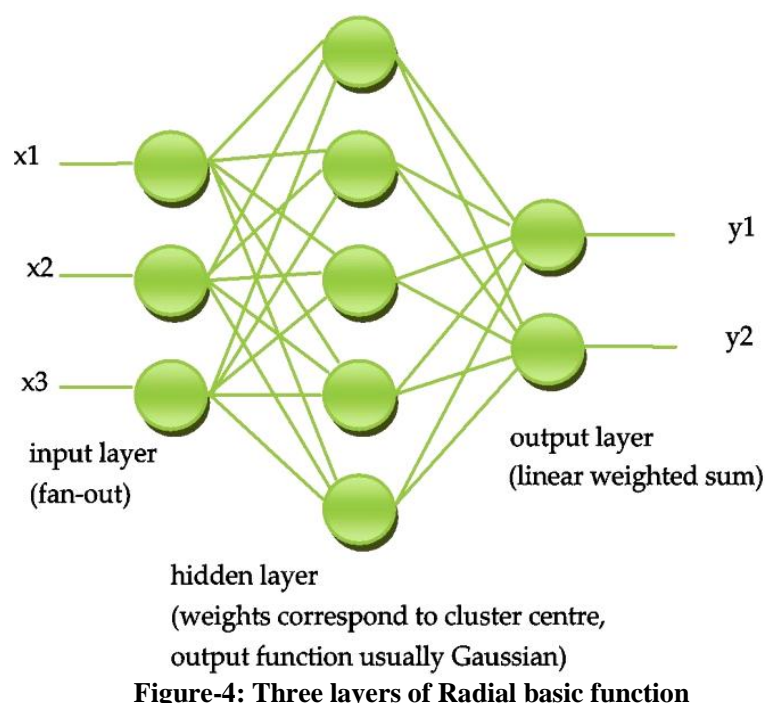

\subsection{Selected Mapping (SLM) Technique}

This method is used for minimization of peak to average transmits power of multicarrier transmission system with selected mapping [17]. Fig.5 shows the block diagram of SLM technique. Here $\mathrm{X}$ represents the input data and $X^{n}$ represents the output data. It generates a set of favorable blocks at the transmitter end which represent the original information and then chooses the most favourable block for transmission as proposed in [18].Here the input block is given by $\mathrm{X}=[\mathrm{X}(0), \mathrm{X}(1), \ldots . . \mathrm{X}(\mathrm{N}-1)]$ is multiplied with $\mathrm{U}$ different phase sequences

$\mathrm{Pu}=[\mathrm{P} 0 \mathrm{u}, \mathrm{P} 1 \mathrm{u}, \ldots . \mathrm{PN}-1 \mathrm{u}]^{\mathrm{T}}$ to produce a modified data block given by

$$
\mathrm{Xu}=[\mathrm{Xu}[1], \mathrm{Xu}[2], \ldots . \mathrm{Xu}[\mathrm{N}-1]]^{\mathrm{T}}
$$

The IFFT of $U$ independent sequences are taken to produce the time domain sequences $\mathrm{xu}=[\mathrm{xu}(0), \mathrm{xu}(1), \ldots . \mathrm{xu}(\mathrm{N}-1)]^{\mathrm{T}}$ among which the only one with the lowest PAPR is selected for transmission. The amount of PAPR reduction for SLM depends on the the proper design of the phase sequences and also on the number of phase sequences U [19]. When using SLM technique the original symbol vector at the receiver end can be recover by transmitting the side band information at the transmitter end. 
U IFFT operations are required for the implementation of SLM technique.

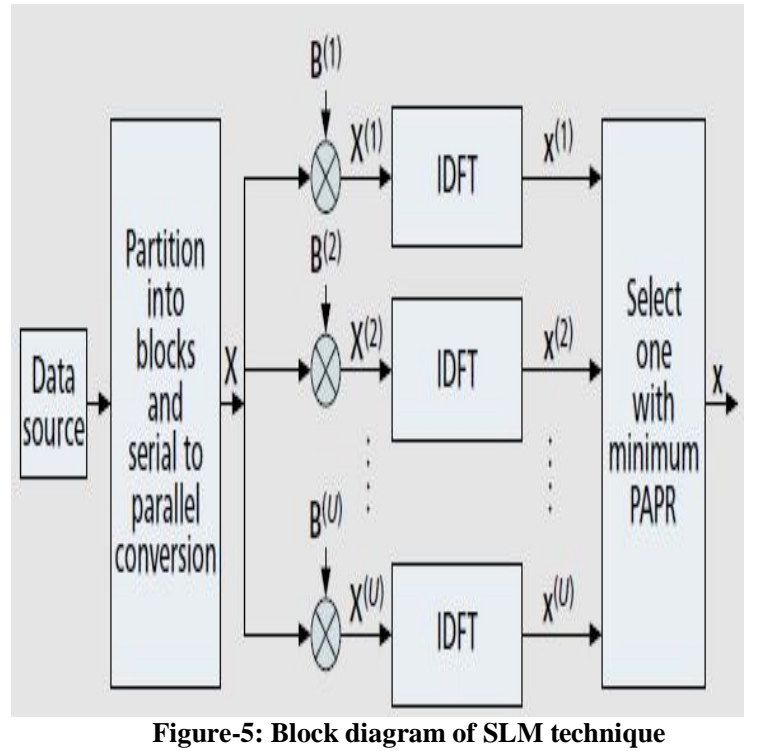

In SLM technique U phase vectors are used for both transmitter and receiver and the index opt of rotation vector as side information with minimum PAPR needs to be transmitted to recover the data sequence $\mathrm{x}$.

\subsection{Tone Reservation}

This method is used for multicarrier transmission and it also shows the reserving tones to reduce the PAPR. This technique includes number of set of reservation of tones. By knowing the number of tones, reserved tones can be used to minimize the PAPR of OFDM signal [20]. This scheme basically depends on the amount of complexity of the signals. If the number of tones is small then reduction in PAPR may represent non negligible samples of available bandwidth. The main advantage of tone reservation is that there is no need to transmit the side information along with the transmitted signal and no process is needed at receiver end and also. In this technique many number of loop are used and the signal will pass from each loop.

\section{CONCLUSION}

The high PAPR (peak-to-average power ratio) of transmitted signals is considered to be one of the major drawbacks of multicarrier transmission because the power efficiency decreases as the signal fluctuation increases. All the potential benefits of OFDM transmission are reduced by high PAPR value. In this paper the conventional PAPR reduction techniques such as tone reservation (TR), clipping , companding transform, radial basic function ( RBF), selected mapping (SLM) technique and partial transmit sequence (PTS) have been discussed . Each technique have some advantage and disadvantage as in cipping bit-error-rate degrades but in tone reservation power increment takes place. For better PAPR reduction performance SLM and PTS techniques provide better performance because in these techniques the input data is divided in to sub-blocks and as the number of sub block increases , PAPR of OFDM signals decreases . In SLM/PTS technique bandwidth also get expanded. For high data rate OFDM system, PAPR reduction technique with low computational complexity can be applied in wireless communication.

\section{REFERENCES}

[1] R. O'neal and L. N. Lopes, "Envelope variation and spectral splatter in clipped multicarrier signals," in Proc. PIMRC'95, Sep. 1995, pp.71-74.

[2] W.Y.Zou and Y.Wu, "COFDM: an overview,"IEEE Trans, Broadcast., vol.41.no.1, pp. 1-7, Mar.1995

[3] J. Tellado and J. M. Cioffi, Multicarrier Modulation with Low PAR, Application to DSL and Wireless. Norwell, MA: Kluwer Academic Publisher, 2000.

[4] Tao Jiang, Yiyan Wu, "An overview of PAPR reduction techniques for OFDM signals",IEEE transactions on Broadcasting,Vol.54,No.2,June 2008.

[5] T. Jiang and Y.W.WU, "An overview: of Peak -to average power ratio reduction techniques for OFDM signals." IEEE Trans. Broadcast., vol. 54, no. 2, pp. 258267, June 2008

[6] S.H.Muller, R.W.Bauml, R.F.H.Fischer, and J.B.Huber, "OFDM with reduced peak to average power ratio by multiple signal representation." Ann. Telecommun., vol.52, no. 1-2, pp. 58-67, feb.1997

[7] J.Tellado and J.M. Cioffi , "PAR reduction in multicarrier transmission systems ," ANSI Document , TIEI.4 Technical Subcommitee, no. 97-367 pp. 1-14, DEC. 8. 1997

[8] M.Sharif,M.Gharivi-Alkghansri and B.H.Khalaj, "On thepeak-to-average power of OFDM signals based on oversampling ," IEEE Trans.commun...vol 51,no.2,pp 7378,jan.2003.

[9] Seok-Joong, Dong-Joon, "A modified SLM scheme with low complexity for PAPR reduction of OFDM systems", IEEE transactions on Broadcasting,Vol.53,No.4,Dec 2007.

[10] X. Wang, T. T. Yjhung, and C. S. Ng, "Reduction of peakto-average power ratio of OFDM system using a companding technique," IEEE Trans. Broadcast., vol. 45, no. 9, pp. 303-307, Sept. 1999.

[11] S. H. Muller, J. B. Huber, "OFDM with Reduced Peak-toAverage Power Ratio by Optimum Combination of Partial Transmit Sequences", Electronics Letters, vol. 33, no. 5, pp. 368 -369, Feb. 1997.

[12] L. J. Cimini, Jr. and N. R. Sollenberger, "Peak-to-average power ratio reduction of an OFDM signal using partial transmit sequences", IEEE Communication Letters., vol. 4, no. 3, pp. 86 - 88, Mar. 2000.

[13] Mukunthan, P. Dananjayan, "Modified PTS with FECs for PAPR Reduction of OFDM Signals," International Journal of Computer Applications (0975 - 8887) Volume 11- No.3, December 2010

[14] B. A. Whitehead and T. D. Choate, "Evolving space-filling curves to distribute radial basis functions over an input space," IEEE Trans. Neural Networks., vol. 5, no. 1, 1994, pp. 15-22.

[15] S. Chen, C. F. N. Cowan, and P. M. Grant, "Orthogonal least squares learning algorithm for radial basis function networks," IEEE Trans. Neural Networks., vol. 2, no. 2, 1991, pp. 303-309' 
[16] U. Mitra and H. V. Poor, "Neural network techniques for adaptive multiuser demodulation," IEEE J Select. Areas Commun., vol. 12, no. 9, Dec. 1994, pp.1461-1469.

[17] Mahmoud Ferdosizadeh Naeiny, "Selected Mapping Algorithm for PAPR Reduction of Space-Frequency Coded OFDM Systems Without Side Information", IEEE transactions on Vehicular technology, vol. 60, no. 3, March 2011.
[18] Seok-Joong, Dong-Joon, "A modified SLM scheme with low complexity for PAPR reduction of OFDM systems", IEEE transactions on Broadcasting,Vol.53,No.4,Dec 2007.

[19] Tao Jiang, Yiyan Wu, "An overview of PAPR reduction techniques for OFDM signals",IEEE transactions on Broadcasting,Vol.54,No.2,June 2008.

[20] Umar Izaz Butt, “A Study On Tone Reservation Technique For Papr Reduction In Ofdm" Year 2010 Dissertation .Com Boca Raton, Florida ISBN-10:1-59942-360-X, ISBN-13: 978-1-59942-360-9 